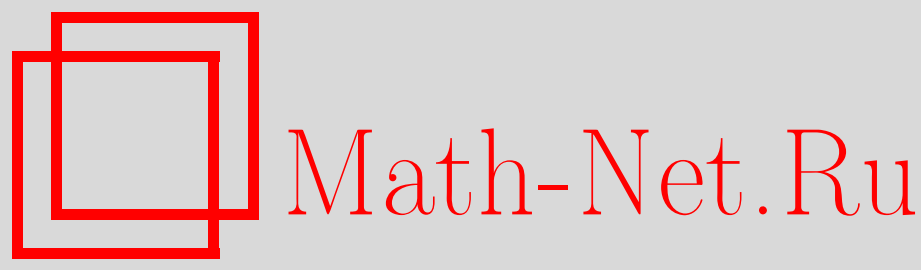

I. Epifani, A. Lijoi, A finitely additive version of the law of the iterated logarithm, Теория вероятн. и ее примен., 1999, том 44, выпуск 4, 757-775

DOI: https://doi.org/10.4213/tvp1064

Использование Общероссийского математического портала Math-Net.Ru подразумевает, что вы прочитали и согласны с пользовательским соглашением

http://www . mathnet.ru/rus/agreement

Параметры загрузки:

IP : 52.87 .193 .239

26 апреля 2023 г., 18:29:08

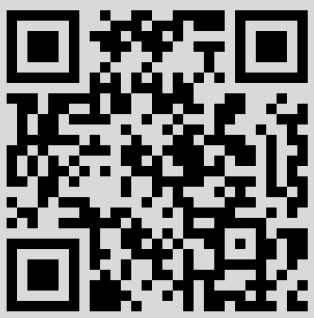




\section{A FINITELY ADDITIVE VERSION OF THE LAW OF THE ITERATED LOGARITHM}

Приводится конечно-аддитивная версия закона повторного логарифма (LIL). В формулировке участвуют только конечномерные распределения последовательности $\left(X_{n}\right)_{n \geqslant 1}$ независимых случайных величин. Доказано, что в случае $\sigma$-аддитивных вероятностных мер полученный результат эквивалентен классическому закону повторного логарифма.

Ключевые слова и фразы: конечно-аддитивная вероятность, закон повторного логарифма, суммы независимых случайных величин.

1. Introduction. When dealing with probabilities that are $\sigma$-additive, one is fundamentally concerned with the problem of measurability of subsets of a given space of elementary events. In this abstract mathematical approach to probability theory, the notion of event is equivalent to that of measurable set.

More realistically, and in the spirit of de Finetti's stance, one might think of an event as something independent of measurability issues. From this viewpoint observability becomes crucial. In other words, probability assessment of an event makes sense only when it is observable.

This remark is quite relevant with respect to limit theorems in probability theory. As an introduction to our work, consider the set $\left\{\lim \sup Z_{n}=c\right\}$, where $\left(Z_{n}\right)_{n \geqslant 1}$ is a sequence of random variables (r.v.'s), and $c$ is a real constant. If $\left(Z_{n}\right)_{n \geqslant 1}$ satisfies some suitable conditions, wellknown, the Law of the Iterated Logarithm (LIL) assigns probability 1 to the previous set. But $\left\{\lim \sup Z_{n}=c\right\}$, though measurable, is not an observable property. This raises the problem of giving alternative formulations of limit theorems, usually presented in the measure-theoretic approach to probability theory.

We proceed in proving a finitistic version of the LIL when one takes into account the possibility of working with probabilities that are not necessarily $\sigma$-additive.

${ }^{*}$ CNR-IAMI, via A. M. Ampère, 56, 20131 Milano, Italy; e-mail: antonio@iami.micnr.it 
The ideas we try to exploit are well expounded in de Finetti [6] and in later contributions by Dubins [7], Zanoni and Regazzini [16]-[18]. They provide finitistic versions of some classical laws of large numbers and useful suggestions for further developments in this field.

As a matter of fact, there is a wide variety of works concerning the study of limit laws for sequences of r.v.'s in the finitely additive setting. However they follow an approach which is different from the one we resort to. Instead of providing formulations of limiting properties in finitistic terms, they aim at giving versions of these properties that are identical to the $\sigma$-additive counterparts but still valid for non $\sigma$-additive probabilities. The key notion in this framework is that of strategy, and guidelines are developed in [8], [7] and [13]. We will not linger on this topic, even though we think that tracing a comparison between the two approaches would be quite a stimulating task.

In what follows, we refer to de Finetti's ideas in order to prove a weak version of the LIL. In Section 2 we describe the finitely additive setting we work with. In particular we clarify the concept of stochastically independent r.v.'s. In Section 3 we prove the main theorem and specify some of its relevant features. In Section 4 it is shown that in the case $\mathbf{P}$ is a $\sigma$-additive probability measure the classical version of the LIL is equivalent to our result. Finally, in Section 5 we illustrate two examples in which the LIL fails, whereas the finitistic law being proposed is applicable.

2. Preliminaries. In this section we introduce some definitions and general results to be used later. Let $\Omega$ be any set of elementary cases, $\mathscr{A}$ an algebra of subsets of $\Omega$ and $\mathbf{P}$ a probability on $(\Omega, \mathscr{A})$. Namely, $\mathbf{P}$ is a non-negative, finitely additive set function on $\Omega$ such that $\mathbf{P}(\Omega)=1$. A Borel-measurable function $X_{n}: \Omega \rightarrow \mathbf{R}(n \geqslant 1)$ will be called random variable (r.v.). Moreover, $\mathscr{B}\left(\mathbf{R}^{n}\right)$ denotes the class of Borel subsets of $\mathbf{R}^{n}$ for all $n \geqslant 1$. In the following sections, we mean by $\mathbf{E} X$ the Dunford-Schwartz integral (see [9, Section III.2], or, alternatively, [1, Section 4.4]). A relevant concept to be introduced for our results is that of stochastic independence. In a finitely additive setting, one may give various definitions of stochastic independence, and in general they are not equivalent (for a deeper insight see $[6$, p. 321-323]). For mathematical convenience, we refer to the following

$\mathrm{D}$ e $\mathrm{f}$ i $\mathrm{n}$ i t i o $\mathrm{n} 1 .\left(X_{n}\right)_{n \geqslant 1}$ is a sequence of stochastically independent r.v.'s on $(\Omega, \mathscr{A}, \mathbf{P})$ if

$$
\begin{aligned}
& \mathbf{P}\{\omega:\left.\left(X_{1}(\omega), \ldots, X_{n}(\omega)\right) \in A_{1},\left(X_{n+1}(\omega), \ldots, X_{n+m}(\omega)\right) \in A_{2}\right\} \\
&= \mathbf{P}\left\{\omega:\left(X_{1}(\omega), \ldots, X_{n}(\omega)\right) \in A_{1}\right\} \\
& \quad \times \mathbf{P}\left\{\omega:\left(X_{n+1}(\omega), \ldots, X_{n+m}(\omega)\right) \in A_{2}\right\}
\end{aligned}
$$

$\forall A_{1} \in \mathscr{B}\left(\mathbf{R}^{n}\right), \forall A_{2} \in \mathscr{B}\left(\mathbf{R}^{m}\right) \forall n, m \geqslant 1$. 
In the last section we give examples of sequences of r.v.'s stochastically independent according to previous definition and governed by a proper finitely additive probability.

Now take $(\Omega, \mathscr{A})$ to be equal to $\left(\mathbf{R}^{\infty}, \mathscr{B}\left(\mathbf{R}^{\infty}\right)\right)$ and $\left(X_{n}\right)_{n \geqslant 1}$ the coordinate process, i.e., $X_{k}(x)=x_{k}, x=\left(x_{1}, x_{2}, \ldots\right) \in \mathbf{R}^{\infty}$. It is possible to define a finitely additive probability $\mathbf{P}$ on $\left(\mathbf{R}^{\infty}, \mathscr{B}\left(\mathbf{R}^{\infty}\right)\right)$ with respect to which the $X_{n}$ 's are stochastically independent according to Definition 1 . The existence of such a $\mathbf{P}$ follows from an analogue of Kolmogorov's extension theorem valid in this more general setting. For a proof of the latter result, the reader may refer to $[5$, p. 41$]$, and $[1$, Section 3.2$]$.

Let us give the following

D e f i n i t i o n 2. Let $\left(Z_{n}\right)_{n \geqslant 1}$ be a sequence of r.v.'s. We say that $b^{*}(n)$ and $b_{*}(n)$ are upper and lower sequences, respectively, for $\left(Z_{n}\right)_{n \geqslant 1}$ if $b^{*}(n)>0, b_{*}(n)>0$ and

(a) $\forall \eta>0 \exists N^{*}=N^{*}(\eta)$ such that $\forall k \geqslant 1$

$$
\mathbf{P}\left\{\bigcap_{n=N}^{N+k}\left\{\frac{Z_{j}}{b^{*}(j)}<1\right\}\right\}>1-\eta \quad \forall N \geqslant N^{*} ;
$$

(b) $\forall \eta>0 \exists N_{*}=N_{*}(\eta)$ and $\forall N \geqslant N_{*}$ and $\forall k \geqslant 1 \exists p_{*}=p_{*}(\eta, N, k)$ such that

$$
\mathbf{P}\left\{\bigcap_{n=N}^{N+k}\left\{\max _{n \leqslant j \leqslant n+p} \frac{Z_{j}}{b_{*}(j)}>1\right\}\right\}>1-\eta \quad \forall p \geqslant p_{*} .
$$

We introduced Definition 2 in order to study the oscillations of a sequence of independent r.v.'s in a finitistic setting. As one may note there is no need to specify any extension of the finite-dimensional distributions of the process $\left(Z_{n}\right)_{n \geqslant 1}$ to $\left(\mathbf{R}^{\infty}, \mathscr{B}\left(\mathbf{R}^{\infty}\right)\right)$. Therefore, our problem is equivalent to the determination, under suitable conditions, of upper and lower sequences for the sequence of partial sums. We will stress this point in Theorem 1 and Theorem 2, and a simple application of these very same theorems provides a finitely additive version of the LIL in Theorem 3 .

3. Main results. In this section we illustrate a version of the LIL which holds in the finitely additive setting. The formulation is strictly related to an article by Cantelli [2], in which the author provides a first extension of Kolmogorov's LIL to a sequence of unbounded r.v.'s, see [10]. Let us specify some notation. $\left(X_{n}\right)_{n \geqslant 1}$ is a sequence of stochastically independent r.v.'s defined on $\left(\mathbf{R}^{\infty}, \mathscr{B}\left(\mathbf{R}^{\infty}\right)\right)$ in the sense of Section 2. Let $\sigma_{n}^{2}=\mathbf{D} X_{n}$, $S_{n}=\sum_{i=1}^{n} X_{i}, s_{n}^{2}=\mathrm{D} S_{n}=\sum_{i=1}^{n} \sigma_{i}^{2}, t_{n}^{2}=2 \log \log s_{n}^{2}, S_{n}^{*}=S_{n} /\left(s_{n} t_{n}\right)$, for all $n \geqslant 1$.

The main assumptions for the validity of our results concern the variance $\sigma_{n}^{2}$ and the absolute moments of order $(2+\delta)$ of the $X_{n}$ 's, for some $\delta \in(0,1]$ : 
(I) $\sigma_{n}^{2} \geqslant \beta>0$ for some constant $\beta$ and for all $n \geqslant 1$,

(II) $\mathbf{E}\left|X_{n}\right|^{2+\delta}<\alpha_{\delta}$ for some $\delta \in(0,1]$, for some $\alpha_{\delta}>0$ and for all $n \geqslant 1$.

We are now in a position to assert the first theorem which specifies the upper sequence.

Theorem 1. Let $\left(X_{n}\right)_{n \geqslant 1}$ be a sequence of independent r.v.'s with zero means for which (I)-(II) hold. Then $\forall \varepsilon>0(1+\varepsilon) s_{n} t_{n}$ is an upper sequence for $\left(S_{n}\right)_{n \geqslant 1}$.

P r o of. Let $\left(n_{i}\right)_{i \geqslant 3}$ be a subsequence in $\mathbf{N}$ defined by $n_{i}:=$ $[\exp \{i / \log i\}]$, where $[x]$ denotes the integer part of $x \in \mathbf{R}$. By resorting to simple probabilistic arguments, the following inequalities can be shown to hold:

$$
\begin{gathered}
\mathbf{P}\left\{\bigcup_{j=\nu}^{\nu+m}\left\{\max _{1 \leqslant n \leqslant n_{j}} S_{n} \geqslant(1+\varepsilon) s_{n_{j-1}} t_{n_{j-1}}\right\}\right\} \\
\leqslant \sum_{j=\nu}^{\nu+m} \mathbf{P}\left\{\max _{1 \leqslant n \leqslant n_{j}} S_{n} \geqslant(1+\varepsilon) s_{n_{j-1}} t_{n_{j-1}}\right\} \\
\leqslant 2^{1+\gamma} \sum_{j=\nu}^{\infty} \mathbf{P}\left\{S_{n_{j}} \geqslant(1+\varepsilon) s_{n_{j-1}} t_{n_{j-1}}-C\right\}
\end{gathered}
$$

for all $\gamma>0$ and for some $C>0$ (for the second inequality above, see Lemma 3.2 in [10]). Moreover, by Lemma 3.1 in [10], $\sum_{j=\nu}^{\infty} \mathbf{P}\left\{S_{n_{j}} \geqslant(1+\right.$ $\left.\varepsilon) s_{n_{j-1}} t_{n_{j-1}}-C\right\}$ is the remainder of a convergent series. Therefore, $\forall \varepsilon>0$ and $\forall \eta>0 \exists \nu_{0}=\nu_{0}(\varepsilon, \eta)$ such that

$$
\mathbf{P}\left\{\bigcup_{j=\nu}^{\nu+m}\left\{\max _{1 \leqslant n \leqslant n_{j}} S_{n} \geqslant(1+\varepsilon) s_{n_{j-1}} t_{n_{j-1}}\right\}\right\}<\eta \quad \forall m \geqslant 1,
$$

or, equivalently, $\mathbf{P}\left\{\cap_{j=\nu}^{\nu+m}\left\{\max _{1 \leqslant n \leqslant n_{j}} S_{n}<(1+\varepsilon) s_{n_{j-1}} t_{n_{j-1}}\right\}\right\}>1-\eta$ for all $m \geqslant 1$. Since $s_{n_{j-1}} t_{n_{j-1}} \leqslant s_{n} t_{n}$ for all $n \in\left\{n_{j-1}, n_{j-1}+1, \ldots, n_{j}\right\}$, one has

$$
\begin{aligned}
& \mathbf{P}\left\{\bigcap_{j=\nu}^{\nu+m} \bigcap_{n=1}^{n_{j}}\left\{S_{n}<(1+\varepsilon) s_{n_{j-1}} t_{n_{j-1}}\right\}\right\} \\
& \quad \leqslant \mathbf{P}\left\{\bigcap_{j=\nu}^{\nu+m} \bigcap_{n=n_{j-1}}^{n_{j}}\left\{S_{n}<(1+\varepsilon) s_{n} t_{n}\right\}\right\}=\mathbf{P}\left\{\bigcap_{j=n_{\nu}}^{n_{\nu+m}}\left\{S_{n}<(1+\varepsilon) s_{n} t_{n}\right\}\right\} .
\end{aligned}
$$

Take $N^{*}=n_{\nu_{0}}$ and $N=n_{\nu}$. For all $k \geqslant 1$ there exists an $m \in \mathbf{N}$ such that $n_{\nu+m}>N+k$. It follows

$$
\mathbf{P}\left\{\bigcap_{n=N}^{N+k}\left\{S_{n}<(1+\varepsilon) s_{n} t_{n}\right\}\right\} \geqslant \mathbf{P}\left\{\bigcap_{j=n_{\nu}}^{n_{\nu+m}}\left\{S_{n}<(1+\varepsilon) s_{n} t_{n}\right\}\right\} .
$$

Theorem 1 is proved. 
Theorem 2. If $\left(X_{n}\right)_{n \geqslant 1}$ is a sequence of independent r.v.'s with zero means satisfying (I)-(II), then $\forall \varepsilon, \eta>0, \exists N_{*}=N_{*}(\varepsilon, \eta)$ and $\forall N \geqslant N_{*}$ $\exists p_{0}=p_{0}(\varepsilon, \eta, N)$ such that

$$
\mathbf{P}\left\{\bigcap_{n=N}^{N+k} \bigcup_{j=n}^{n+p}\left\{S_{j}^{*}>1-\varepsilon\right\}\right\}>1-\eta
$$

$\forall k \geqslant 1, \quad \forall p \geqslant \max \left\{p_{0}, e^{k}\right\}$.

It is possible to split the proof of Theorem 2 in three steps.

In the first part we need to work with independent events. Thus, we give a result for the differences of partial sums along a convenient subsequence of positive integers. The second step consists in passing from the subsequence of differences to the subsequence of partial sums. Finally, we get the assertion over the whole sequence by reductio ad absurdum.

The first step is characterized by the following lemma.

Lemma 1. Let $\left(n_{i}\right)_{i \geqslant 3}$ be a subsequence of positive integers defined by $n_{i}:=[\exp \{i \log \log i\}]$ and $(*):=\left\{j_{1}, \ldots, j_{l+1} \in\left\{n_{\nu+1}, n_{\nu+2}, \ldots, n_{\nu+m}\right\}:\right.$ $\left.j_{1}<\cdots<j_{l+1}\right\}$. Then $\forall \varepsilon, \eta>0 \exists \nu_{0}=\nu_{0}(\varepsilon, \eta)$ and $\forall \nu \geqslant \nu_{0} \exists m_{0}=$ $m_{0}(\varepsilon, \eta, \nu)$ such that

$$
\mathbf{P}\left\{\bigcup_{(*)}^{l+1}\left\{\bigcap_{r=2}-S_{j_{r}}>(1-\varepsilon) s_{j_{r}} t_{j_{r}}\right\}\right\}>1-\eta
$$

$\forall l \geqslant 1, \forall m \geqslant \max \left\{m_{0}, 2^{l}\right\}$.

P r o of. To simplify notation, let

$$
A_{j_{r}}^{(\varepsilon)}:=\left\{S_{j_{r}}-S_{j_{r-1}}>(1-\varepsilon) s_{j_{r}} t_{j_{r}}\right\}, \quad C_{\nu, m, l}^{\varepsilon}:=\bigcap_{(*)}^{l+1} \bigcup_{r=2}^{(\varepsilon)^{c}}
$$

We can interpret $C_{\nu, m, l}^{\varepsilon}$ as the event which is true if and only if at most $l-1$ of the $m$ events $A_{n_{i}}^{(\varepsilon)}(i=\nu+1, \ldots, \nu+m)$ are verified. Hence, if $B_{\nu, m}^{(j)}$ is the event corresponding to exactly $j$ of the $A_{n_{i}}^{(\varepsilon)}$ 's $(i=\nu+1, \ldots, \nu+m)$ being verified,

$$
\mathbf{P}\left(C_{\nu, m, l}^{\varepsilon}\right)=\sum_{j=0}^{l-1} \mathbf{P}\left(B_{\nu, m}^{(j)}\right)
$$

We try to estimate $\mathbf{P}\left(B_{\nu, m}^{(j)}\right)$. If $j=0$,

$$
\begin{aligned}
\mathbf{P}\left(B_{\nu, m}^{(0)}\right) & =\mathbf{P}\left\{\bigcap_{i=\nu+1}^{\nu+m} A_{n_{i}}^{(\varepsilon)^{c}}\right\}=\prod_{i=\nu+1}^{\nu+m}\left(1-\mathbf{P}\left(A_{n_{i}}^{(\varepsilon)}\right)\right) \\
& \leqslant \exp \left\{-\sum_{i=\nu+1}^{\nu+m} p_{n_{i}}\right\} \quad\left[p_{n_{i}}:=\mathbf{P}\left(A_{n_{i}}^{(\varepsilon)}\right)\right] .
\end{aligned}
$$


For any other $j \in\{1, \ldots, l-1\}$, we define (口) $:=\left\{s_{1}, \ldots, s_{j} \in\right.$ $\left.\left\{n_{\nu+1}, \ldots, n_{\nu+m}\right\}, s_{1}<\cdots<s_{j}\right\}$ and $q_{j+1}, \ldots, q_{m} \in\left\{n_{\nu+1}, \ldots, n_{\nu+m}\right\} \backslash$ $\left\{s_{1}, \ldots, s_{j}\right\}$, so that

$$
\begin{aligned}
\mathbf{P}\left(B_{\nu, m}^{(j)}\right) & =\sum_{(\square)} p_{s_{1}} \cdots p_{s_{j}} \prod_{i=j+1}^{m}\left(1-p_{q_{i}}\right) \leqslant \sum_{(\square)} p_{s_{1}} \cdots p_{s_{j}} \exp \left\{-\sum_{i=j+1}^{m} p_{q_{i}}\right\} \\
& =\exp \left\{-\sum_{i=\nu+1}^{\nu+m} p_{n_{i}}\right\} \sum_{(\square)} p_{s_{1}} \cdots p_{s_{j}} \exp \left\{\sum_{i=1}^{j} p_{s_{i}}\right\} \\
& =\exp \left\{-\sum_{i=\nu+1}^{\nu+m} p_{n_{i}}\right\} \sum_{(\square)} \sum_{(* *)} \frac{1}{j !} p_{\gamma_{1}} \cdots p_{\gamma_{j}} \exp \left\{\sum_{i=1}^{j} p_{\gamma_{i}}\right\}
\end{aligned}
$$

where $(* *)$ stands for the set of all permutations $\gamma_{1}, \ldots, \gamma_{j}$ of indices $s_{1}, \ldots, s_{j}$.

Moreover, $\sum_{(\square)} \sum_{(* *)} p_{\gamma_{1}} \cdots p_{\gamma_{j}}=\sum_{(+)} p_{\gamma_{1}} \cdots p_{\gamma_{j}}$, where the latter sum is extended over all $j$-permutations of a set of $m$ elements. Thus

$$
\begin{aligned}
\mathbf{P}\left(B_{\nu, m}^{(j)}\right) \leqslant & \exp \left\{-\sum_{i=\nu+1}^{\nu+m} p_{n_{i}}\right\} \frac{1}{j !} \sum_{(+)} p_{\gamma_{1}} e^{p_{\gamma_{1}}} \cdots p_{\gamma_{j}} e^{p_{\gamma_{j}}} \\
\leqslant & \exp \left\{-\sum_{i=\nu+1}^{\nu+m} p_{n_{i}}\right\} \frac{1}{j !}\left(\sum_{i=\nu+1}^{\nu+m} p_{n_{i}} e^{p_{n_{i}}}\right)^{j} \\
= & \exp \left\{-\sum_{i=\nu+1}^{\nu+m} p_{n_{i}}\left(1-e^{p_{n_{i}}}\right)\right\} \frac{1}{j !}\left(\sum_{i=\nu+1}^{\nu+m} p_{n_{i}} e^{p_{n_{i}}}\right)^{j} \\
& \times \exp \left\{-\left(\sum_{i=\nu+1}^{\nu+m} p_{n_{i}} e^{p_{n_{i}}}\right)\right\}, \\
\mathbf{P}\left(C_{\nu, m, l}^{\varepsilon}\right)= & \sum_{j=0}^{l-1} \mathbf{P}\left(B_{\nu, m}^{(j)}\right) \leqslant \exp \left\{-\sum_{i=\nu+1}^{\nu+m} p_{n_{i}}\left(1-e^{p_{n_{i}}}\right)\right\} \\
& \times \sum_{j=0}^{l-1} \frac{1}{j !}\left(\sum_{i=\nu+1}^{\nu+m} p_{n_{i}} e^{p_{n_{i}}}\right)^{j} \exp \left\{-\sum_{i=\nu+1}^{\nu+m} p_{n_{i}} e^{p_{n_{i}}}\right\} .
\end{aligned}
$$

In order to estimate the expression in (3), the following inequalities for $p_{n_{i}}$ are valid.

With $\left(n_{i}\right)_{i \geqslant 3}$ as above, for every $\varepsilon>0, \gamma>0$ there exist $i_{0}=i_{0}(\varepsilon, \gamma)$ and $C_{j}=C_{j}\left(\varepsilon, \gamma, \delta, \beta, \alpha_{\delta}\right)(j=1,2)$ such that $\forall i \geqslant i_{0}$

$$
C_{1}\left(\frac{1}{i \log \log i}\right)^{(1+\gamma)(1-\varepsilon)^{2}} \leqslant p_{n_{i}} \leqslant C_{2}\left(\frac{1}{i \log \log i}\right)^{(1-\gamma)(1-\varepsilon)^{2}} .
$$

The required estimate of $p_{n_{i}}$ is achieved by applying a Large Deviations type result. Proceeding in a similar fashion as in Theorem 7.1.3 in [3, p. 203], 
one can prove that if $\left(n_{i}\right)_{i \geqslant 1}$ is such that $n_{i}-n_{i-1} \sim n_{i} \quad(i \rightarrow \infty)$, and if

$$
z_{n_{i}}:=\log \frac{\sum_{j=n_{i-1}+1}^{n_{i}} \mathbf{E}\left|X_{j}\right|^{2+\delta}}{s_{n_{i}}^{2+\delta}}+(1+\gamma)(1-\varepsilon)^{2} \frac{s_{n_{i-1}}^{2} t_{n_{i-1}}^{2}}{2\left(s_{n_{i}}^{2}-s_{n_{i-1}}^{2}\right)} \longrightarrow-\infty
$$

as $i \rightarrow \infty$ for all $\varepsilon>0$ and for all $\gamma>0$, then there is $i^{*}=i^{*}(\varepsilon, \gamma)$ such that

$$
\begin{aligned}
& \exp \left\{-(1+\gamma)(1-\varepsilon)^{2} \frac{s_{n_{i}}^{2} t_{n_{i}}^{2}}{2\left(s_{n_{i}}^{2}-s_{n_{i-1}}^{2}\right)}\right\} \leqslant p_{n_{i}} \\
& \quad \leqslant \exp \left\{-(1-\gamma)(1-\varepsilon)^{2} \frac{s_{n_{i}}^{2} t_{n_{i}}^{2}}{2\left(s_{n_{i}}^{2}-s_{n_{i-1}}^{2}\right)}\right\}
\end{aligned}
$$

for all $i \geqslant i^{*}$.

When $n_{i}=[\exp \{i \log \log i\}]$, it is easily verified that $n_{i}-n_{i-1} \sim n_{i}$ and, under our assumptions (I)-(II), (5) is valid. Indeed,

$$
\beta^{2+\delta}<\left(\sigma_{n}^{2}\right)^{2+\delta} \leqslant\left(\mathbf{E}\left|X_{n}\right|^{2+\delta}\right)^{2}<\alpha_{\delta}^{2}
$$

and

$$
\begin{aligned}
\log \left\{\frac{\sum_{j=n_{i-1}+1}^{n_{i}} \mathbf{E}\left|X_{j}\right|^{2+\delta}}{s_{n_{i}}^{2+\delta}}\right\}+(1+\gamma)(1-\varepsilon)^{2} \frac{s_{n_{i}}^{2} t_{n_{i}}^{2}}{2\left(s_{n_{i}}^{2}-s_{n_{i-1}}^{2}\right)} \\
<\log \frac{\left(n_{i}-n_{i-1}\right) \alpha_{\delta}}{\left(n_{i}-n_{i-1}\right)^{1+\delta / 2} \beta^{1+\delta / 2}} \\
\quad+(1+\gamma)(1-\varepsilon)^{2} \frac{n_{i} \alpha_{\delta}^{2 /(2+\delta)} 2}{2\left(n_{i}-n_{i-1}\right) \beta} \log \log \left(n_{i} \alpha_{\delta}^{2 /(2+\delta)}\right) \\
=\log \frac{\alpha_{\delta}}{\left(n_{i}-n_{i-1}\right)^{\delta / 2} \beta^{1+\delta / 2}} \\
\quad+(1+\gamma)(1-\varepsilon)^{2} \frac{n_{i} \alpha_{\delta}^{2 /(2+\delta)}}{\left(n_{i}-n_{i-1}\right) \beta} \log \log \left(n_{i} \alpha_{\delta}^{2 /(2+\delta)}\right) \\
\sim-\frac{\delta}{2} \log \left(n_{i}-n_{i-1}\right)+(1+\gamma)(1-\varepsilon)^{2} \frac{\alpha_{\delta}^{2 /(2+\delta)}}{\beta} \log \log n_{i} \longrightarrow-\infty
\end{aligned}
$$

By (I)-(II), for every $i$ there is a $\kappa_{i} \in\left(\beta, \alpha_{\delta}^{2 /(2+\delta)}\right)$ such that $s_{n_{i}}^{2}=\kappa_{i} n_{i}$, and, therefore,

$$
\frac{s_{n_{i}}^{2} t_{n_{i}}^{2}}{2\left(s_{n_{i}}^{2}-s_{n_{i-1}}^{2}\right)} \sim \frac{1}{i \log \log i} \quad \text { as } \quad i \rightarrow \infty
$$

and this completes the proof of (4) if we choose a suitable $i_{0}=i_{0}(\varepsilon, \gamma)>i^{*}$.

Now, for sufficiently small $\varepsilon>0$ one can determine a $\gamma=\gamma(\varepsilon)>0$ such that $(1+\gamma)(1-\varepsilon)^{2}<1$ and $2(1-\gamma)(1-\varepsilon)^{2}>1$. These two conditions imply $\sum_{i=3}^{\infty} p_{n_{i}}=\infty$ and $\sum_{i=3}^{\infty} p_{n_{i}}^{2}<\infty$.

In other words, we can now write in $(4) i_{0}=i_{0}(\varepsilon)$. 
Turning back to (3), the first term in the product, $\exp \left\{-\sum_{i=\nu+1}^{\nu+m} p_{n_{i}}(1-\right.$ $\left.\left.e^{p_{n_{i}}}\right)\right\}$, is bounded for every $\nu, m \geqslant 1$. To see this, note that $-p_{n_{i}}(1-$ $\left.e^{p_{n_{i}}}\right) \sim p_{n_{i}}^{2}($ as $i \rightarrow \infty)$. We shall denote by $K$ a constant such that $\exp \left\{-\sum_{i=\nu+1}^{\nu+m} p_{n_{i}}\left(1-e^{p_{n_{i}}}\right)\right\} \leqslant K$.

As far as the second term in (3) is concerned, let $\xi_{i}(i=\nu+1, \ldots, \nu+m)$ be a random variable distributed according to a Poisson law with parameter $p_{n_{i}} e^{p_{n_{i}}}$ and let the $\xi_{i}$ 's be stochastically independent. Then,

$$
\sum_{j=0}^{l-1} \frac{1}{j !}\left(\sum_{i=\nu+1}^{\nu+m} p_{n_{i}} e^{p_{n_{i}}}\right)^{j} \exp \left\{-\sum_{i=\nu+1}^{\nu+m} p_{n_{i}} e^{p_{n_{i}}}\right\}=\mathbf{P}\left\{\sum_{i=\nu+1}^{\nu+m} \xi_{i} \leqslant l-1\right\} \text {. }
$$

Moreover, by the Berry-Esseen inequality (cf. [12, p. 115]), we get

$$
\begin{aligned}
\mathbf{P}\left\{\sum \xi_{i} \leqslant l-1\right\}= & \mathbf{P}\left\{\sum \xi_{i} \leqslant l-1\right\}-\Phi\left(\frac{l-1-\sum p_{n_{i}} e^{p_{n_{i}}}}{\sqrt{\sum p_{n_{i}} e^{p_{n_{i}}}}}\right) \\
& +\Phi\left(\frac{l-1-\sum p_{n_{i}} e^{p_{n_{i}}}}{\sqrt{\sum p_{n_{i}} e^{p_{n_{i}}}}}\right) \\
\leqslant & A \frac{\sum \mathbf{E}\left|\xi_{i}\right|^{3}}{b_{\nu, m}^{3 / 2}}+\Phi\left(\frac{l-1-\sum p_{n_{i}} e^{p_{n_{i}}}}{\sqrt{\sum p_{n_{i}} e^{p_{n_{i}}}}}\right)
\end{aligned}
$$

where all sums are taken with $i$ running from $\nu+1$ up to $\nu+m, b_{\nu, m}:=$ $\sum_{i=\nu+1}^{\nu+m} \mathbf{D}\left(\xi_{i}\right)=\sum_{i=\nu+1}^{\nu+m} p_{n_{i}} e^{p_{n_{i}}}, A>0$ is an appropriate constant and $\Phi(\cdot)$ is the standard Gaussian distribution function. Obviously

$$
\begin{aligned}
\frac{\sum_{i=\nu+1}^{\nu+m} \mathbf{E}\left|\xi_{i}\right|^{3}}{b_{\nu, m}^{3 / 2}} & =\frac{\sum_{i=\nu+1}^{\nu+m}\left(p_{n_{i}} e^{p_{n_{i}}}+3 p_{n_{i}}^{2} e^{2 p_{n_{i}}}+p_{n_{i}}^{3} e^{3 p_{n_{i}}}\right)}{\left(\sum_{i=\nu+1}^{\nu+m} p_{n_{i}} e^{p_{n_{i}}}\right)^{3 / 2}} \\
& \sim \frac{\sum_{i=\nu+1}^{\nu+m} p_{n_{i}} e^{p_{n_{i}}}}{\left(\sum_{i=\nu+1}^{\nu+m} p_{n_{i}} e^{p_{n_{i}}}\right)^{3 / 2}} \\
& \sim \frac{1}{\left(\sum_{i=\nu+1}^{\nu+m} p_{n_{i}} e^{p_{n_{i}}}\right)^{1 / 2}} \longrightarrow 0, \quad m \rightarrow \infty .
\end{aligned}
$$

As far as the second summand in (8) is concerned, $\Phi$, we let $\mathscr{D}:=\{(m, l) \in$ $\left.\mathbf{N}^{2}: m \geqslant 2^{l}\right\}$. This implies

$$
\Phi\left(\frac{l-1-\sum p_{n_{i}} e^{p_{n_{i}}}}{\sqrt{\sum p_{n_{i}} e^{p_{n_{i}}}}}\right) \leqslant \Phi\left(\frac{\log _{2} m-\sum p_{n_{i}} e^{p_{n_{i}}}}{\sqrt{\sum p_{n_{i}} e^{p_{n_{i}}}}}\right) \quad \forall(m, l) \in \mathscr{D} .
$$

We now try to estimate the right-hand side in the previous inequality. By Euler's summation formula (see [11, p. 521]), one obtains, with $\mu=(1+\gamma)(1-\varepsilon)^{2}$,

$$
\begin{aligned}
\sum_{i=\nu+1}^{\nu+m} p_{n_{i}} & \geqslant \sum_{i=\nu+1}^{\nu+m}\left(\frac{1}{i \log \log i}\right)^{\mu} \sim \int_{\nu+1}^{\nu+m}\left(\frac{1}{x \log \log x}\right)^{\mu} d x \quad \text { as } m \rightarrow \infty \\
& =\int_{\log (\nu+1)}^{\log (\nu+m)} \frac{e^{(1-\mu) t}}{(\log t)^{\mu}} d t
\end{aligned}
$$




$$
\begin{aligned}
\geqslant & \frac{1}{1-\mu} e^{(1-\mu) \log (\nu+m)} \frac{1}{(\log \log (\nu+m))^{\mu}} \\
& -\frac{1}{1-\mu} e^{(1-\mu) \log (\nu+1)} \frac{1}{(\log \log (\nu+1))^{\mu}} \quad \text { [integrate by parts] } \\
\sim & \frac{1}{1-\mu} \cdot \frac{m^{(1-\mu)}}{(\log \log m)^{\mu}} \quad \text { as } m \rightarrow \infty .
\end{aligned}
$$

Since $p_{n_{i}} \leqslant p_{n_{i}} e^{p_{n_{i}}}$ for all $i$

$$
\frac{\log _{2} m-\sum p_{n_{i}} e^{p_{n_{i}}}}{\sqrt{\sum p_{n_{i}} e^{p_{n_{i}}}}} \leqslant \frac{\log _{2} m-(1-\mu)^{-1} m^{(1-\mu)}(\log \log m)^{-\mu}}{\sqrt{(1-\mu)^{-1} m^{(1-\mu)}(\log \log m)^{-\mu}}} \rightarrow-\infty
$$

as $m \rightarrow \infty$ and

$$
\Phi\left(\frac{l-1-\sum_{i=\nu+1}^{\nu+m} p_{n_{i}} e^{p_{n_{i}}}}{\sqrt{\sum_{i=\nu+1}^{\nu+m} p_{n_{i}} e^{p_{n_{i}}}}}\right) \rightarrow 0
$$

Finally, for all $(m, l) \in \mathscr{D}$

$$
\begin{aligned}
\mathbf{P}\left(C_{\nu, m, l}^{\varepsilon}\right) & \leqslant \exp \left\{-\sum_{i=\nu+1}^{\nu+m} p_{n_{i}}\left(1-e^{p_{n_{i}}}\right)\right\} \mathbf{P}\left\{\sum_{i=\nu+1}^{\nu+m} \xi_{i} \leqslant l-1\right\} \\
& \leqslant K \mathbf{P}\left\{\sum_{i=\nu+1}^{\nu+m} \xi_{i} \leqslant \log _{2} m\right\} .
\end{aligned}
$$

Therefore, for any $\varepsilon, \eta>0$ there is $\nu_{0}=\nu_{0}(\varepsilon, \eta) \geqslant i_{0}(\varepsilon)$ and for all $\nu \geqslant \nu_{0}$ one can find $m_{0}=m_{0}(\varepsilon, \eta, \nu) \in \mathbf{N}$ such that $\mathbf{P}\left(C_{\nu, m, l}^{\varepsilon}\right)<\eta \forall m \geqslant \max \left\{m_{0}, 2^{l}\right\}$.

Taking into account the definition of the event $C_{\nu, m, l}^{\varepsilon}$

$$
\mathbf{P}\left\{\bigcup_{(*)}^{l+1}\left\{\bigcap_{r=2}\left\{S_{j_{r}}-S_{j_{r-1}}>(1-\varepsilon) s_{j_{r}} t_{j_{r}}\right\}\right\}>1-\eta\right.
$$

and Lemma 1 is finally proved.

Next, we aim at passing from the subsequence of differences of partial sums to the subsequence of partial sums. Lemma 2 below provides the result we need.

Lemma 2. Let $\left(n_{i}\right)_{i \geqslant 3}$ be the subsequence of positive integers defined in Lemma 1. Then for any $\varepsilon, \eta>0$ there is a $\nu_{00}=\nu_{00}(\varepsilon, \eta)>0$ and for any $\nu \geqslant \nu_{00}$ there is a strictly positive integer $m_{00}=m_{00}(\varepsilon, \eta, \nu)$ such that

$$
\mathbf{P}\left\{\bigcap_{h=\nu+1}^{\nu+l}\left\{\max _{h \leqslant j \leqslant h+m} \frac{S_{n_{j}}}{s_{n_{j}} t_{n_{j}}}>1-\varepsilon\right\}\right\}>1-2 \eta
$$

for all $m \geqslant \max \left\{m_{00} ; 2^{l}\right\}$.

$\mathrm{Pr}$ o of. Since

$$
\bigcap_{h=\nu+1}^{\nu+l} \bigcup_{j=h}^{h+m}\left\{S_{n_{j}}>(1-\varepsilon) s_{n_{j}} t_{n_{j}}\right\} \supset \bigcup_{(*)}^{l+1}\left\{\bigcap_{r=2}^{l+1}\left\{S_{j_{r}}>(1-\varepsilon) s_{j_{r}} t_{j_{r}}\right\}=: D_{\nu, m, l}^{\varepsilon}\right.
$$


it is sufficient to prove the assertion for $D_{\nu, m, l}^{\varepsilon}$. One may note that

$$
\begin{gathered}
\mathbf{P}\left\{\bigcup_{(*)}^{l+1}\left\{\bigcap_{r=2}\left\{(1-\varepsilon) s_{j_{r}} t_{j_{r}}-(1+\varepsilon) s_{j_{r-1}} t_{j_{r-1}}\right\}\right\}\right. \\
\geqslant \mathbf{P}\left\{\bigcup_{(*)}^{l+1}\left\{\bigcap_{r=2}-S_{j_{r-1}}>(1-\varepsilon) s_{j_{r}} t_{j_{r}}\right\}\right. \\
\geqslant \mathbf{P}\left\{\bigcup_{(*)}^{l+1}\left\{\bigcap_{j_{r-1}}>-(1+\varepsilon) s_{j_{r-1}} t_{j_{r-1}}\right\}\right\} \\
\left.\bigcap S_{j_{r}}-S_{j_{r-1}}>(1-\varepsilon) s_{j_{r}} t_{j_{r}}\right\} \\
\left.\bigcap_{i=1}^{m}\left\{S_{n_{\nu+i}}<(1+\varepsilon) s_{n_{\nu+i}} t_{n_{\nu+i}}\right\}\right\}>1-2 \eta
\end{gathered}
$$

because of $(2)$ and Theorem 1 applied to the sequence $\left(-X_{n}\right)_{n \geqslant 1}$. Since for a sufficiently large $r$ there exists $\varepsilon^{\prime}>\varepsilon$ such that $(1-\varepsilon) s_{j_{r}} t_{j_{r}}-$ $(1+\varepsilon) s_{j_{r-1}} t_{j_{r-1}}>\left(1-\varepsilon^{\prime}\right) s_{j_{r}} t_{j_{r}}$, then

$$
\mathbf{P}\left\{\bigcup_{(*)}^{l+1}\left\{\bigcap_{r=2}\left\{S_{j_{r}}>(1-\varepsilon) s_{j_{r}} t_{j_{r}}\right\}\right\}>1-2 \eta .\right.
$$

Lemma 2 is proved.

$\mathrm{P} \mathrm{r}$ o of of $\mathrm{T} \mathrm{h}$ e or e $\mathrm{m} 2$. By virtue of Lemma 2, we only need to extend (10) to the whole sequence. We proceed by reductio ad absurdum. Suppose (1) does not hold. Then for some $\varepsilon, \eta>0$, for every $N_{*}, p_{0} \in \mathbf{N}$ there are $\tilde{N} \geqslant N_{*}, \widetilde{k}=\tilde{k}\left(p_{0}, N_{*}\right)$ and $p^{*} \geqslant \max \left\{p_{0}, e^{\widetilde{k}}\right\}$ such that

$$
1-\eta>\mathbf{P}\left\{\bigcap_{n=\widetilde{N}}^{\tilde{N}+\widetilde{k}} \bigcup_{j=n}^{n+p^{*}}\left\{S_{j}^{*}>1-\varepsilon\right\}\right\} \geqslant \mathbf{P}\left\{\bigcup_{j=\widetilde{N}+\widetilde{k}}^{\widetilde{N}+p^{*}}\left\{S_{j}^{*}>1-\varepsilon\right\}\right\} .
$$

Choose $\eta^{*}$ such that $2 \eta^{*}<\eta$. Then we can find a $\nu_{00}=\nu_{00}\left(\varepsilon, \eta^{*}\right)$ and $\forall \nu \geqslant \nu_{00}$ there is a $m_{00}=m_{00}\left(\varepsilon, \eta^{*}, \nu\right)$ such that for every $l \in \mathbf{N}$, and $m \geqslant \max \left\{m_{00}, 2^{l}\right\}$ (10) holds with $\eta^{*}$ in place of $\eta$. Observe that

$$
\begin{gathered}
\bigcap_{r=\nu+1}^{\nu+l} \bigcup_{j=r}^{r+m}\left\{S_{n_{j}}^{*}>1-\varepsilon\right\} \subset \bigcap_{r=1}^{l-1} \bigcap_{j=n_{\nu+r}}^{n_{\nu+r+1}} \bigcup_{i=j}^{n_{\nu+m+r+1}}\left\{S_{i}^{*}>1-\varepsilon\right\} \\
\subset \bigcap_{j=n_{\nu+1}}^{n_{\nu+2}} \bigcup_{i=j}^{n_{\nu+m+2}}\left\{S_{i}^{*}>1-\varepsilon\right\} \subset \bigcup_{i=n_{\nu+2}}^{n_{\nu+m+2}}\left\{S_{i}^{*}>1-\varepsilon\right\} .
\end{gathered}
$$

For $\widetilde{N}$ and $\widetilde{k}$ fixed above, it is possible to find a $\nu \in \mathbf{N}\left(\nu>\nu_{00}\right)$ such that $\tilde{N}+\widetilde{k} \leqslant n_{\nu+2}=[\exp \{(\nu+2) \log \log (\nu+2)\}]$, i.e., $\tilde{k} \leqslant \exp \{(\nu+$ 
2) $\log \log (\nu+2)\}-\tilde{N}-1$. If we also find out that $\tilde{N}+p^{*} \geqslant n_{\nu+m+2}=$ $[\exp \{(\nu+m+2) \log \log (\nu+m+2)\}]$, then we would have

$$
\bigcup_{n=\widetilde{N}+\widetilde{k}}^{\widetilde{N}+p^{*}}\left\{S_{n}^{*}>1-\varepsilon\right\} \supset \bigcup_{j=n_{\nu+2}}^{n_{\nu+m+2}}\left\{S_{j}^{*}>1-\varepsilon\right\} .
$$

Since $p^{*}>e^{\widetilde{k}}$, it is sufficient that $\tilde{N}+e^{\widetilde{k}} \geqslant \exp \{(\nu+m+2) \log \log (\nu+m+2)\}$, which means $\widetilde{k}>\log (\exp \{(\nu+m+2) \log \log (\nu+m+2)\}-\tilde{N})=(\nu+m+$ 2) $\log \log (\nu+m+2)+\log \left(1-\tilde{N} e^{-(\nu+m+2) \log \log (\nu+m+2)}\right)$. Hence, (11) holds if

$$
\begin{aligned}
z_{\nu} & =(\nu+m+2) \log \log (\nu+m+2)+\log \left(1-\tilde{N} e^{-(\nu+m+2) \log \log (\nu+m+2)}\right) \\
& \leqslant \widetilde{k} \leqslant \exp \{(\nu+2) \log \log (\nu+2)\}-\widetilde{N}-1=y_{\nu}
\end{aligned}
$$

and these inequalities are consistent for a sufficiently large $\nu \geqslant \nu_{00}$. In the case $z_{\nu}>\tilde{k}$

$$
\bigcup_{n=\widetilde{N}+\widetilde{k}}^{\widetilde{N}+p^{*}}\left\{S_{n}^{*}>1-\varepsilon\right\} \supset \bigcup_{n=\widetilde{N}+\left[z_{\nu}\right]}^{\widetilde{N}+p^{*}}\left\{S_{n}^{*}>1-\varepsilon\right\} \supset \bigcup_{j=n_{\nu+2}}^{n_{\nu+m+2}}\left\{S_{j}^{*}>1-\varepsilon\right\} .
$$

Finally, as a consequence of our hypotheses we would have

$$
1-\eta>\mathbf{P}\left\{\bigcup_{n=\widetilde{N}+\widetilde{k}}^{\widetilde{N}+p^{*}}\left\{S_{n}^{*}>1-\varepsilon\right\}\right\} \geqslant \mathbf{P}\left\{\bigcup_{j=n_{\nu+2}}^{n_{\nu+m+2}}\left\{S_{j}^{*}>1-\varepsilon\right\}\right\}>1-2 \eta^{*}
$$

and this is absurd because $2 \eta^{*}<\eta$. Theorem 2 is proved.

Joint application of the results in Theorem 1 and Theorem 2 provides a finitistic characterization of the oscillations problem for the sequence $\left(S_{n}\right)_{n \geqslant 1}$.

Theorem 3. Let $\left(X_{n}\right)_{n \geqslant 1}$ be a sequence of independent r.v.'s with zero means. If assumptions (I)-(II) are satisfied, then $\forall \varepsilon, \eta>0, \exists N_{0}=$ $N_{0}(\varepsilon, \eta) \in \mathbf{N}$, and $\forall N \geqslant N_{0} \exists p_{0}=p_{0}(\varepsilon, \eta, N)$ such that

$$
\mathbf{P}\left\{\bigcap_{n=N}^{N+k}\left\{\left|\max _{n \leqslant j \leqslant n+p} \frac{S_{j}}{s_{j} t_{j}}-1\right|<\varepsilon\right\}\right\}>1-\eta
$$

$\forall k \geqslant 1, \forall p \geqslant \max \left\{p_{0}, e^{k}\right\}$.

P r o o f. Let us put

$$
\begin{aligned}
A_{1}^{(\varepsilon)}(N, p, k) & :=\bigcap_{n=N}^{N+k}\left\{\max _{n \leqslant j \leqslant n+p} S_{j}^{*}<1+\varepsilon\right\}, \\
A_{2}^{(\varepsilon)}(N, p, k) & :=\bigcap_{n=N}^{N+k}\left\{\max _{n \leqslant j \leqslant n+p} S_{j}^{*}>1-\varepsilon\right\} .
\end{aligned}
$$

In order to prove (12) one can show that for every $\varepsilon>0$ and $\eta>0$ there is a strictly positive integer $N_{0}=N_{0}(\varepsilon, \eta)$ and for every $N \geqslant N_{0}$ there exists a 
$p_{0}=p_{0}(\varepsilon, \eta, N) \in \mathbf{N}$ such that, if $p^{*}=\max \left\{p_{0}, e^{k}\right\}$

$$
\begin{aligned}
& \mathbf{P}\left(A_{1}^{(\varepsilon)}(N, p, k)\right)>1-\frac{\eta}{2} \quad \forall N \geqslant N_{0}, \forall k \geqslant 1, \forall p \geqslant p^{*}, \\
& \mathbf{P}\left(A_{2}^{(\varepsilon)}(N, p, k)\right)>1-\frac{\eta}{2} \quad \forall N \geqslant N_{0}, \forall k \geqslant 1, \forall p \geqslant p^{*} .
\end{aligned}
$$

But, clearly, if $N_{0}=\max \left\{N^{*}, N_{*}\right\}$ (see Theorem 1 and Theorem 2), then Theorem 1 entails (13), whereas (1) implies (14). Theorem 3 is proved.

$\mathrm{R}$ e mark 1. As a matter of fact, (12) does not completely characterize oscillations of partial sums on finitary sets. We have to look at the behaviour of $\min S_{n}^{*}$ as well.

Corollary 1. Suppose hypotheses (I)-(II) are satisfied. Then $\forall \varepsilon, \eta>0$ $\exists N_{0}=N_{0}(\varepsilon, \eta) \in \mathbf{N}$ and $\forall N \geqslant N_{0} \exists p_{0}=p_{0}(\varepsilon, \eta, N) \in \mathbf{N}$ such that

$$
\mathbf{P}\left\{\bigcap_{n=N}^{N+k}\left\{\left|\min _{n \leqslant j \leqslant n+p} \frac{S_{j}}{s_{j} t_{j}}+1\right|<\varepsilon\right\}\right\}>1-\eta
$$

$\forall k \geqslant 1, \forall p \geqslant \max \left\{p_{0}, e^{k}\right\}$.

$\mathrm{P} \mathrm{r}$ o of. One may proceed exactly as in the proof of (12) using the sequence $\left(-X_{n}\right)_{n \geqslant 1}$ instead of $\left(X_{n}\right)_{n \geqslant 1}$, and observing that $\min S_{j}^{*}=$ $-\max \left(-S_{j}^{*}\right)$.

$\mathrm{R}$ e m a rk 2. A natural question to be raised in our framework regards uniqueness of the upper and lower sequences obtained in Theorem 3. More precisely, it is worth investigating the possibility of existence of a r.v. $Y$ (defined on the same probability space as the $X_{n}$ 's) such that $\bar{b}(\varepsilon, n)=(Y+\varepsilon) s_{n} t_{n}$ and $\underline{b}(\varepsilon, n)=(Y-\varepsilon) s_{n} t_{n}$ are also upper and lower sequences. The answer requires the notion of equivalence.

$\mathrm{D}$ e f in ition 3. Two r.v.'s, $X$ and $Y$, are called equivalent, write $X \simeq Y$, if $\forall \varepsilon>0 \mathbf{P}\{|X-Y|<\varepsilon\}=1$.

Notice that, unlike the $\sigma$-additive setting, previous definition does not imply $\mathbf{P}\{X=Y\}=1$ (see [18] for some illustrative examples).

Proposition 1. Suppose (12) holds. Then $\forall \varepsilon, \eta>0, \exists N_{0}=N_{0}(\varepsilon, \eta)$, and $\forall N \geqslant N_{0} \exists p_{0}=p_{0}(\varepsilon, \eta, N)$ such that

$$
\mathbf{P}\left\{\bigcap_{n=N}^{N+k}\left\{\left|\max _{n \leqslant j \leqslant n+p} S_{j}^{*}-Y\right|<\varepsilon\right\}\right\}>1-\eta \quad \forall k \geqslant 1, \forall p \geqslant \max \left\{p_{0}, e^{k}\right\}
$$

if and only if $Y \simeq 1$.

P r o o f. Suppose $Y$ is a r.v. for which (16) holds. We need to show (i) $\mathbf{P}\{Y>1+\varepsilon\}=0$ and (ii) $\mathbf{P}\{Y<1-\varepsilon\}=0$. Observe that trivially

$$
\begin{aligned}
\mathbf{P}\{Y>1+\varepsilon\}= & \mathbf{P}\left\{\bigcap_{n=N}^{N+k} \bigcup_{j=n}^{n+p}\left\{S_{j}^{*}>Y-\frac{\varepsilon}{2}\right\} \bigcap\{Y>1+\varepsilon\}\right\} \\
& +\mathbf{P}\left\{\bigcup_{n=N}^{N+k} \bigcap_{j=n}^{n+p}\left\{S_{j}^{*} \leqslant Y-\frac{\varepsilon}{2}\right\} \bigcap\{Y>1+\varepsilon\}\right\}
\end{aligned}
$$


and

$$
\{Y>1+\varepsilon\} \equiv\left\{Y-\frac{\varepsilon}{2}>1+\frac{\varepsilon}{2}\right\}
$$

Thus, by (16),

$$
\mathbf{P}\{Y>1+\varepsilon\}<\mathbf{P}\left\{\bigcap_{n=N}^{N+k} \bigcup_{j=n}^{n+p}\left\{S_{j}^{*}>1+\frac{\varepsilon}{2}\right\}\right\}+\frac{\eta}{2}<\eta .
$$

Proceeding in a similar fashion

$$
\mathbf{P}\{Y<1-\varepsilon\}<\mathbf{P}\left\{\bigcap_{n=N}^{N+k}\left\{S_{n}^{*}<1-\frac{\varepsilon}{2}\right\}\right\}+\frac{\eta}{2}<\eta .
$$

The arbitrariness of $\eta>0$ provides the result we were to prove.

Conversely, suppose $Y \simeq 1$. Let us choose $\varepsilon>0$ and $\delta \in(0, \varepsilon)$. If we fix $\eta>0$ then there exists $N_{0}=N_{0}(\varepsilon, \eta)$ such that for all $N \geqslant N_{0}$ and for all $k \geqslant 1$

$$
\begin{aligned}
1-\eta & <\mathbf{P}\left\{\bigcap_{n=N}^{N+k}\left\{S_{n}^{*}<1+(\varepsilon-\delta)\right\}\right\} \\
& =\mathbf{P}\left\{\bigcap_{n=N}^{N+k}\left\{S_{n}^{*}<1+(\varepsilon-\delta)\right\} \bigcap\{Y>1-\delta\}\right\} \\
& \leqslant \mathbf{P}\left\{\bigcap_{n=N}^{N+k}\left\{S_{n}^{*}<Y+\varepsilon\right\}\right\} .
\end{aligned}
$$

On the other hand

$$
\begin{aligned}
1-\eta & <\mathbf{P}\left\{\bigcap_{n=N}^{N+k} \bigcup_{j=n}^{n+p}\left\{S_{j}^{*}>1-(\varepsilon-\delta)\right\}\right\} \\
& =\mathbf{P}\left\{\bigcap_{n=N}^{N+k} \bigcup_{j=n}^{n+p}\left\{S_{j}^{*}>1-(\varepsilon-\delta)\right\} \bigcap\{Y<1+\delta\}\right\} \\
& \leqslant \mathbf{P}\left\{\bigcap_{n=N}^{N+k} \bigcup_{j=n}^{n+p}\left\{S_{j}^{*}>Y-\varepsilon\right\}\right\} .
\end{aligned}
$$

Proposition 1 is proved.

4. Connections with the $\sigma$-additive case. We shall look more carefully at (12) in order to explain why it can be considered as a finitistic version of the LIL. As it is well known, in the $\sigma$-additive setting lower and upper sequences for the partial sums of independent r.v.'s are provided by the LIL. In the case $\mathbf{P}$ is $\sigma$-additive, our result (12) implies the LIL. Conversely, it is also possible to prove that if the LIL holds, then an analogue to (12) is easily deduced. We will better specify this point below. 
Proposition 2. Let $\mathbf{P}$ be a probability measure. Then

$$
\mathbf{P}\left\{\lim \sup S_{n}^{*}=1\right\}=1
$$

if and only if $\forall \varepsilon, \eta>0, \forall k \geqslant 1 \exists N_{0}=N_{0}(\varepsilon, \eta)$, and $\forall N \geqslant N_{0} \exists p_{0}=$ $p_{0}(\varepsilon, \eta, N, k)$ such that

$$
\mathbf{P}\left\{\bigcap_{n=N}^{N+k}\left\{\left|\max _{n \leqslant j \leqslant n+p} S_{j}^{*}-1\right|<\varepsilon\right\}\right\}>1-\eta \quad \forall p \geqslant p_{0} .
$$

$\mathrm{P} \mathrm{r}$ o o f. If $\mathbf{P}$ is $\sigma$-additive, (17) holds if and only if

$$
\mathbf{P}\left\{S_{n}^{*}<1+\varepsilon \text { for all but finitely many } n\right\}=1 \quad \forall \varepsilon>0
$$

and

$$
\mathbf{P}\left\{S_{n}^{*}>1-\varepsilon \text { infinitely often }\right\}=1 \quad \forall \varepsilon>0 .
$$

Now, (18a) can be written as $\mathbf{P}\left\{\cup_{n=1}^{\infty} \cap_{j=n}^{\infty}\left\{S_{j}^{*}<1+\varepsilon\right\}\right\}=1$ that is $\mathbf{P}\left\{\lim _{n \rightarrow \infty} \cap_{j=n}^{\infty}\left\{S_{j}^{*}<1+\varepsilon\right\}\right\}=1$. By the continuity of $\mathbf{P}$, we have $\lim _{n} \mathbf{P}\left\{\cap_{j=n}^{\infty}\left\{S_{j}^{*}<1+\varepsilon\right\}\right\}=1$. This entails that $\forall \varepsilon, \eta>0, \exists N_{0}=N_{0}(\varepsilon, \eta)$ such that

$$
\mathbf{P}\left\{\bigcap_{n=N}^{N+k}\left\{S_{n}^{*}<1+\varepsilon\right\}\right\}>1-\eta \quad \forall N \geqslant N_{0}, \forall k \geqslant 1 .
$$

On the other hand, (18b) is tantamount to $\mathbf{P}\left\{\cap_{n=1}^{\infty} \cup_{j=n}^{\infty}\left\{S_{j}^{*}>1-\varepsilon\right\}\right\}=1$. Let us denote by $D_{j}^{(n)}(j>n)$ the event

$$
\left\{S_{m}^{*}<1-\varepsilon, m=n, \ldots, j-1, \text { and } S_{j}^{*}>1-\varepsilon\right\}
$$

whereas $D_{n}^{(n)}=\left\{S_{n}^{*}>1-\varepsilon\right\}$. For a given $n$, the events $\left\{D_{j}^{(n)}: j=\right.$ $n, n+1, \ldots\}$ are disjoint and $\cup_{j=n}^{\infty} D_{j}^{(n)}=\cup_{j=n}^{\infty}\left\{S_{j}^{*}>1-\varepsilon\right\}$. As usual, $\mathbf{I}_{A}$ is the indicator function of $A,(18 \mathrm{~b})$ is rewritten as follows

$$
\mathbf{E}\left(\prod_{n=1}^{\infty} \sum_{j=n}^{\infty} \mathbf{I}_{D_{j}^{(n)}}\right)=1
$$

A fortiori, $\mathbf{E}\left(\prod_{n=N}^{N+k} \sum_{j=n}^{\infty} \mathbf{I}_{D_{j}^{(n)}}\right)=1 \forall N \geqslant 1, \forall k \geqslant 1$ and, consequently,

$$
\mathbf{E}\left(\lim _{p \rightarrow \infty} \prod_{n=N}^{N+k} \sum_{j=n}^{n+p} \mathbf{I}_{D_{j}^{(n)}}\right)=1 .
$$

Since $\prod_{n=N}^{N+k} \sum_{j=n}^{n+p} \mathbf{I}_{D_{j}^{(n)}}$ is a non-decreasing sequence in $p$, by the monotone convergence theorem

$$
\lim _{p \rightarrow \infty} \mathbf{E}\left(\prod_{n=N}^{N+k} \sum_{j=n}^{n+p} \mathbf{I}_{D_{j}^{(n)}}\right)=1
$$


which means that $\forall \varepsilon, \eta>0, \forall k \geqslant 1, \forall N \geqslant 1, \exists p_{0}=p_{0}(\varepsilon, \eta, N, k)$ such that

$$
\mathbf{E}\left(\prod_{n=N}^{N+k} \sum_{j=n}^{n+p} \mathbf{I}_{D_{j}^{(n)}}\right)>1-\eta \quad \forall p \geqslant p_{0} .
$$

If we express the latter in terms of the probability $\mathbf{P}$ we reach the result. Indeed

$$
\begin{aligned}
\mathbf{E}\left(\prod_{n=N}^{N+k} \sum_{j=n}^{n+p} \mathbf{I}_{D_{j}^{(n)}}\right) & =\mathbf{E}\left(\mathbf{I}_{\bigcap_{n=N}^{N+k} \bigcup_{j=n}^{n+p} D_{j}^{(n)}}\right)=\mathbf{E}\left(\mathbf{I}_{\bigcap \bigcup\left\{S_{j}^{*}>1-\varepsilon\right\}}\right) \\
& =\mathbf{P}\left\{\bigcap_{n=N}^{N+k} \bigcup_{j=n}^{n+p}\left\{S_{j}^{*}>1-\varepsilon\right\}\right\} .
\end{aligned}
$$

Conversely, suppose that $\forall \varepsilon, \eta>0, \exists N_{0}=N_{0}(\varepsilon, \eta)$ such that

$$
\mathbf{P}\left\{\bigcap_{n=N}^{N+k}\left\{S_{j}^{*}<1+\varepsilon\right\}\right\}>1-\eta \quad \forall N \geqslant N_{0}, \forall k \geqslant 1 .
$$

By continuity of $\mathbf{P}$ we get $\mathbf{P}\left\{\cap_{n=N}^{\infty}\left\{S_{n}^{*}<1+\varepsilon\right\}\right\}>1-\eta$ which clearly implies (18a).

Moreover if $\forall \varepsilon, \eta>0 \exists N_{0}=N_{0}(\varepsilon, \eta)$ and $\forall N \geqslant N_{0} \exists p_{0}=p_{0}(\varepsilon, \eta, N)$ such that $\mathbf{P}\left\{\cap_{n=N}^{N+k}\left\{\max _{n \leqslant j \leqslant n+p} S_{j}^{*}>1-\varepsilon\right\}\right\}>1-\eta \forall k \geqslant 1$ and $\forall p \geqslant$ $\max \left\{p_{0}, e^{k}\right\}$, a fortiori

$$
\mathbf{P}\left\{\bigcap_{n=N}^{N+k}\left\{\max _{j \geqslant n} S_{j}^{*}>1-\varepsilon\right\}\right\}>1-\eta .
$$

Again, by continuity of $\mathbf{P}$, if we take the limit as $k \rightarrow \infty$ we have $\mathbf{P}\left\{\cap_{n=N}^{\infty} \cup_{j=n}^{\infty}\left\{S_{j}^{*}>1-\varepsilon\right\}\right\}>1-\eta$. But the latter implies, by virtue of the arbitrariness in $\eta,(18 \mathrm{~b})$. Proposition 2 is proved.

This very same proposition allows us to say that (12) implies, in a $\sigma$-additive setting, the LIL. Actually (12) contains an even stronger information, in the sense it provides an estimate of $p_{0}(\varepsilon, \eta, N, k)$ in $k$, since we found that $p_{0}(\varepsilon, \eta, N, k)>e^{k}$.

5. Two illustrative examples. In this section we illustrate a couple of examples which do not allow the application of the $\sigma$-additive version of the LIL. On the other hand, it is meaningful to study the oscillations of partial sums in a finitistic setting according to previous Theorem 3 and Corollary 1. This also underlines the fact that (12) and (17) are equivalent only if $\mathbf{P}$ is completely additive.

E x a m p l e 1 . Let $\Omega:=\{1,2, \ldots\}$ and $\mathscr{A}=\mathscr{P}(\Omega)$, the power set of $\Omega$. We define on $(\Omega, \mathscr{P}(\Omega))$ the following probability measure, for every $n \geqslant 1$,

$$
\mathbf{P}_{n}(E):=\frac{1}{n} \#(\omega \in \Omega: 1 \leqslant \omega \leqslant n, \omega \in E)
$$


for all $E \in \mathscr{P}(\Omega)$ and $\# B$ denotes the cardinality of the set $B$. A finitely additive probability, $\mathbf{P}$, is obtained via the rule

$$
\mathbf{P}(E)=\lim _{n \rightarrow \infty} \mathbf{P}_{n}(E)
$$

for all $E$ such that previous limit exists. Let $\mathscr{L}$ be the class of events for which this limit exists. Consider, now, the set of prime numbers and its ordering in $p_{0}, p_{1}, p_{2}, \ldots\left(p_{0}=1, p_{1}=2, p_{2}=3, \ldots\right)$ and associate to each $\omega \in \Omega$ its factorization in prime numbers $\omega=\prod_{r=1}^{\infty} p_{r}^{\alpha_{r}(\omega)}$ where $\alpha_{r}(\omega)$ stands for the exact power of $p_{r}$ in the factorization of $\omega$. If $\omega$ is not divisible by $p_{r}$, then $\alpha_{r}(\omega)=0$. As a consequence, only a finite number of $\alpha_{r}$ 's in the previous representation are different from zero. In this framework, let $\left(X_{n}\right)_{n \geqslant 1}$ be a sequence of r.v.'s on $(\Omega, \mathscr{L}, \mathbf{P})$ defined by

$$
X_{i}(\omega)= \begin{cases}1, & \omega \in[0]_{p_{i}}, \\ 0, & \omega \notin[0]_{p_{i}},\end{cases}
$$

and $[0]_{p_{i}}$ is the residue class of 0 modulo $p_{i}$. It is easy to prove that

$$
\mathbf{P}_{n}\left\{\omega: X_{i}(\omega)=1\right\}=\frac{1}{n}\left[\frac{n}{p_{i}}\right] \quad \forall i \geqslant 1 \forall n \geqslant 1,
$$

and immediately one obtains $\mathbf{P}\left\{\omega: X_{i}(\omega)=1\right\}=1 / p_{i} \quad \forall i \geqslant 1$. By virtue of the additivity of the $\mathbf{P}_{n}$ 's

$$
\mathbf{P}_{n}\left\{\omega: X_{i}(\omega)=0\right\}=1-\mathbf{P}_{n}\left\{\omega: X_{i}(\omega)=1\right\} \underset{n \rightarrow \infty}{\longrightarrow} 1-\frac{1}{p_{i}} .
$$

Hence, $X_{i}$ is a r.v. distributed according to a Bernoulli law with parameter $1 / p_{i}$. Take, now, a pair of r.v.'s $X_{i}, X_{j}$ (for all $i \not \equiv j$ ) and notice that

$$
\begin{aligned}
\mathbf{P}_{n}\left\{\omega: X_{i}(\omega)=1, X_{j}(\omega)=1\right\} & =\frac{1}{n}\left[\frac{n}{p_{i} p_{j}}\right] \underset{n \rightarrow \infty}{\longrightarrow} \frac{1}{p_{i} p_{j}} \\
& =\mathbf{P}\left\{\omega: X_{i}(\omega)=1, X_{j}(\omega)=1\right\} .
\end{aligned}
$$

Moreover

$$
\begin{aligned}
& \mathbf{P}_{n}\left\{\omega: X_{i}(\omega)=0, X_{j}(\omega)=1\right\} \\
& \quad=\mathbf{P}_{n}\left\{\omega: X_{j}(\omega)=1\right\}-\mathbf{P}_{n}\left\{\omega: X_{i}(\omega)=1, X_{j}(\omega)=1\right\} \\
& \underset{n \rightarrow \infty}{\longrightarrow} \frac{1}{p_{j}}\left(1-\frac{1}{p_{i}}\right)=\mathbf{P}\left\{\omega: X_{i}(\omega)=0, X_{j}(\omega)=1\right\}
\end{aligned}
$$

and

$$
\begin{aligned}
& \mathbf{P}_{n}\left\{\omega: X_{i}(\omega)=0, X_{j}(\omega)=0\right\} \\
& \quad=\mathbf{P}_{n}\left\{\omega: X_{i}(\omega)=0\right\}-\mathbf{P}_{n}\left\{\omega: X_{i}(\omega)=0, X_{j}(\omega)=1\right\} \\
& \quad \underset{n \rightarrow \infty}{\longrightarrow}\left(1-\frac{1}{p_{i}}\right)\left(1-\frac{1}{p_{j}}\right)=\mathbf{P}\left\{\omega: X_{i}(\omega)=0, X_{j}(\omega)=0\right\}
\end{aligned}
$$


In a similar fashion, one may prove that

$$
\mathbf{P}\left\{\omega: X_{1}(\omega)=x_{1}, \ldots, X_{k}(\omega)=x_{k}\right\}=\prod_{i=1}^{k}\left(\frac{1}{p_{i}}\right)^{x_{i}}\left(1-\frac{1}{p_{i}}\right)^{1-x_{i}}
$$

for every $\left(x_{1}, \ldots, x_{k}\right) \in\{0,1\}^{k}$ and $k \geqslant 1$. Therefore, $\left(X_{n}\right)_{n \geqslant 1}$ is a sequence of stochastically independent r.v.'s, in accordance with Definition 2. It is clear that $S_{n}(\omega)=\sum_{j=1}^{n} X_{j}(\omega)$ is bounded for every $\omega \in \Omega$ and $n \geqslant 1$. Furthermore, since it is known that $p_{j} \asymp j \log j, \mu_{n}:=\mathbf{E} S_{n}=$ $\sum_{j=1}^{n}\left(1 / p_{j}\right) \longrightarrow \infty, n \rightarrow \infty, s_{n}^{2}=\sum_{j=1}^{n}\left(1 / p_{j}\right)\left(1-\left(1 / p_{j}\right)\right)$ and

$$
\left\{\omega: \lim _{n \rightarrow+\infty} \frac{S_{n}(\omega)-\mu_{n}}{\sqrt{2 s_{n}^{2} \log \log s_{n}^{2}}}=-\infty\right\}=\Omega \text {. }
$$

Consequently, the classical version of the LIL does not hold. On the other hand, since assumptions of Theorem 3 are satisfied, it is possible to characterize the oscillating behaviour of $S_{n}$ on finitary sets in the following terms: $\forall \varepsilon, \eta>0 \exists N_{0}=N_{0}(\varepsilon, \eta)$ and $\forall N \geqslant N_{0} \exists p_{0}=p_{0}(\varepsilon, \eta, N)$ such that $\forall k \geqslant 1 \forall p \geqslant \max \left\{p_{0}, e^{k}\right\}$

$$
\begin{aligned}
& \mathbf{P}\left\{\bigcap_{n=N}^{N+k}\left\{\left|\max _{n \leqslant j \leqslant n+p} \frac{S_{j}-\mu_{j}}{\sqrt{2 s_{n}^{2} \log \log s_{n}^{2}}}-1\right|<\varepsilon\right\}\right\}>1-\eta, \\
& \mathbf{P}\left\{\bigcap_{n=N}^{N+k}\left\{\left|\min _{n \leqslant j \leqslant n+p} \frac{S_{j}-\mu_{j}}{\sqrt{2 s_{n}^{2} \log \log s_{n}^{2}}}+1\right|<\varepsilon\right\}\right\}>1-\eta .
\end{aligned}
$$

An example, analogous to the one just illustrated, was given by de Finetti [4] and it was later rediscovered, independently, by Ramakrishnan and Sudderth [14]. They aim at showing that the $\sigma$-additive law of large numbers may fail in the finitely additive setting.

E x a m p l e 2. Let $\pi(\cdot)$ be a probability measure defined by $\pi(X=$ $x)=e^{-1} / x !, x=0,1,2, \ldots$, and take $Q_{1}\left(X_{1}=x_{1}, \ldots, X_{m}=x_{m}\right)=$ $e^{-m} / \prod_{j=1}^{m}\left(x_{j} !\right)$. Let us now introduce, for some strictly positive integer $M$, the probability

$$
\begin{aligned}
& Q\left\{X_{1}=x_{1}, \ldots, X_{m}=x_{m}\right\} \\
& :=\frac{Q_{1}\left\{X_{1}=x_{1}, \ldots, X_{m}=x_{m}, X_{1} \leqslant M, \ldots, X_{m} \leqslant M\right\}}{Q_{1}\left(X_{1} \leqslant M, \ldots, X_{m} \leqslant M\right)} \\
& \quad=\prod_{j=1}^{m} \frac{\pi\left\{X_{j}=x_{j} ; X_{j} \leqslant M\right\}}{\pi\left(X_{j} \leqslant M\right)}=: \prod_{j=1}^{m} Q\left\{X_{j}=x_{j}\right\},
\end{aligned}
$$

where $Q(X=x)=(1 / x !)\left(\sum_{y=0}^{M} 1 / y !\right)^{-1}, x=0,1, \ldots, M$. Let $\mathscr{C}$ the algebra generated by the cylinders of $\mathbf{N}^{\infty}$ with measurable and finite dimensional basis. Let $\sigma(\mathscr{C})$ be the $\sigma$-algebra generated by $\mathscr{C}$ and $P_{1}$ the $\sigma$-additive extension of $Q_{1}$ to $\sigma(\mathscr{C})$. If

$$
\mathbf{P}(E):=\lim _{m \rightarrow+\infty} \frac{P_{1}\left\{E \bigcap \bigcap_{j=1}^{m}\left\{X_{j} \leqslant M\right\}\right\}}{Q_{1}\left\{\bigcap_{j=1}^{m}\left\{X_{j} \leqslant M\right\}\right\}}
$$


is defined on the class $\mathscr{L}=\{E: \mathbf{P}(E)$ exists $\}$, then $\mathscr{L} \subset \sigma(\mathscr{C})$ and $\mathbf{P}$ is a probability on $\mathscr{L}$ (see [15]). Moreover, $\mathbf{P}(E)=Q(E), \forall E \in \mathscr{C}$. Indeed, one observes that $\mathscr{C} \subset \mathscr{L}$, since $\forall E \in \mathscr{C}$

$$
\mathbf{P}(E)=\lim _{m \rightarrow+\infty} \frac{Q_{1}\left\{E \bigcap \bigcap_{j=1}^{m}\left\{X_{j} \leqslant M\right\}\right\}}{Q_{1}\left\{\bigcap_{j=1}^{m}\left\{X_{j} \leqslant M\right\}\right\}}=\lim _{m \rightarrow \infty} Q(E)=Q(E) .
$$

The r.v.'s $X_{1}, \ldots, X_{n}, \ldots$ are independent with respect to $\mathbf{P}$. In fact, for every $k$-tuple $n_{1}, \ldots, n_{k}$ of integers one has

$$
\begin{aligned}
\mathbf{P}\left\{X_{n_{1}}=x_{1}, \ldots, X_{n_{k}}=x_{k}\right\} & =Q\left\{X_{n_{1}}=x_{1}, \ldots, X_{n_{k}}=x_{k}\right\} \\
& =\prod_{j=1}^{k} Q\left\{X_{n_{j}}=x_{j}\right\}=\prod_{j=1}^{k} \mathbf{P}\left\{X_{n_{j}}=x_{j}\right\} .
\end{aligned}
$$

By straightforward algebra, we obtain the following expressions for the mean and the variance of the $X_{n}$ 's

$$
\begin{aligned}
& \mathbf{E} X=1-\frac{1}{M ! \sum_{x=0}^{M} 1 / x !}=: \mu_{M}, \\
& \mathbf{D} X=1-\frac{1}{M ! \sum_{x=0}^{M} 1 / x !}\left(M-\frac{1}{M ! \sum_{x=0}^{M} 1 / x !}\right)=: \sigma_{M}^{2} .
\end{aligned}
$$

It is clear that $0<\mu_{M}<1$. We shall verify $\left\{\lim \sup \left(S_{n}-n \mu_{M}\right) /\right.$ $\left.\sqrt{2 n \sigma_{M}^{2} \log \log \sigma_{M}^{2}}=1\right\} \in \mathscr{L}$. In order to show it, write

$$
\begin{aligned}
\frac{S_{n}-n \mu_{M}}{\sqrt{2 n \sigma_{M}^{2} \log \log {\sigma_{M}^{2}}^{2}}=} & \frac{S_{n}-n}{\sqrt{2 n \log \log n}} \cdot \sqrt{\frac{\log \log n}{\log \log n \sigma_{M}^{2}}} \cdot \frac{1}{\sigma_{M}} \\
& +\frac{n\left(1-\mu_{M}\right)}{\sqrt{2 n \sigma_{M}^{2} \log \log \sigma_{M}^{2}}}=A_{n}+B_{n} .
\end{aligned}
$$

Trivially, $B_{n} \rightarrow+\infty \quad(n \rightarrow \infty)$. Moreover, $\liminf \left(S_{n}-n\right) / \sqrt{2 n \log \log n}=$ $-1 \quad P_{1}$-a.s. But $\limsup \left(A_{n}+B_{n}\right) \geqslant \liminf A_{n}+\lim B_{n}=+\infty P_{1}$-a.s. Therefore

$$
P_{1}\left\{\limsup \frac{S_{n}-n \mu_{M}}{\sqrt{2 n \sigma_{M}^{2} \log \log \sigma_{M}^{2}}}=1\right\}=0 .
$$

By taking into account the definition of $\mathbf{P}$

$$
\mathbf{P}\left\{\limsup \frac{S_{n}-n \mu_{M}}{\sqrt{2 n \sigma_{M}^{2} \log \log \sigma_{M}^{2}}}=1\right\}=0 .
$$

Thus $\left\{\lim \sup \left(S_{n}-n \mu_{M}\right) / \sqrt{2 n \sigma_{M}^{2} \log \log \sigma_{M}^{2}}=1\right\} \in \mathscr{L}$ and the LIL does not hold.

If we, instead, consider the problem in a finitistic setting, we notice that the hypotheses of Theorem 3 are verified with respect to $\mathbf{P}$. Again, by means of (12) we can describe, in finitistic terms, the oscillations of the $S_{n}$ 's. 
Acknowledgements. We are grateful to Professor E. Regazzini for his enlightening suggestions which helped us in developing this research.

\section{REFERENCES}

1. Bhaskara Rao K. P. S., Bhaskara Rao M. - Theory of Charges. A Study of Finitely Additive Measures. London: Academic Press, 1983, 315 p.

2. Cantelli F.P. Considerazioni sulla legge uniforme dei grandi numeri e sulla generalizzazione di un fondamentale teorema del sig. Paul Lévy. - Giorn. Istit. Ital. Attuari, 1933, v. 4, p. 321-350.

3. Chung K.L. A Course in Probability Theory. New York: Academic Press, 1974, $365 \mathrm{p}$.

4. De Finetti $B$. Sui passaggi al limite nel calcolo delle probabilità. - Rend. Istit. Lomb. Sci. Lett., 1930, v. 63, p. 155-166.

5. De Finetti $B$. Sull'impostazione assiomatica del calcolo delle probabilità. - Ann. Triestini, 1949, v. 19, sez. II, p. 29-81. (English transl.: De Finetti B. Probability, Induction and Statistics. Ch. 5. New York: Wiley, 1972, 266 p.

6. de Finetti $B$. Teoria delle probabilità. V. 1. Torino: Einaudi, 1970. (English transl.: de Finetti $B$. Theory of Probability. New York: Wiley, 1990, 375 p.)

7. Dubins L.E. On Lebesgue-like extensions of finitely additive measures. - Ann. Probab., 1974, v. 2, p. 456-463.

8. Dubins L.E., Savage L.J. How to gamble if you must. New York: McGraw-Hill, 1965. See also: Dubins L. E., Savage L.J. Inequalities for stochastic processes. New York: Dover, 1976, $251 \mathrm{p}$.

9. Dunford N., Schwartz J.T. Linear Operators. Part I: General Theory. New York: Wiley, 1988, $858 \mathrm{p}$.

10. Epifani I., Lijoi A. Some considerations on a version of the Law of the Iterated Logarithm due to F. P. Cantelli. Technical Report 95-21. Milan: CNR-IAMI, 1995. To appear in Rend. Istit. Lomb. Sci. Lett.

11. Knopp K. Theory and Application of Infinite Series. New York: Dover, 1990.

12. Petrov V. V. Sums of Independent Random Variables. Berlin: Springer-Verlag, 1975, $345 \mathrm{p}$.

13. Purves R.A., Sudderth W.D. Some finitely additive probability. - Ann. Probab., 1976 , v. 4, p. 259-276.

14. Ramakrishnan S., Sudderth W.D. A sequence of coin toss variables for which the strong law fails. - Amer. Math. Monthly, 1988, v. 95, p. 939-941.

15. Regazzini E. De Finetti's coherence and statistical inference. - Ann. Statist., 1987, v. 15, p. $845-864$.

16. Regazzini E. Observability and probabilistic limit theorems. - International Conference «The Notion of Event in Probabilistic Epistemology». Trieste, 27-29 May 1996.

17. Regazzini E., Zanoni G. Stochastic convergence in a finitely additive setting. Quaderno 29/1988. Dip. to Mat. «F. Enriques». Milano: Univ. Studi di Milano, 1988.

18. Zanoni $G$. Successioni di variabili aleatorie dotate di leggi di probabilità finitamente additive. Degree Thesis. Dip. to Mat. «F. Enriques», Milano: Univ. Studi di Milano, 1987. 